\title{
EL DIARIO PERSONAL EN FRANCIA A PRINCIPIOS DEL SIGLO XXI: INFLEXIONES DE UN GÉNERO LITERARIO ${ }^{1}$
}

\author{
THE DIARY IN FRANCE \\ AT THE BEGINNING OF THE 21TH CENTURY: \\ INFLECTIONS OF A LITERARY GENRE
}

\author{
Michel BRAUD \\ Universidad de Pau et des Pays de l'Adour \\ michel.braud@univ-pau.fr
}

\begin{abstract}
Resumen: Aparecido en Francia a finales del siglo XVIII en el ámbito privado, el diario personal se impone progresivamente en el curso de los siglos XIX y XX como un género literario para conocer diversas mutaciones a finales del siglo XX. La banalización del género, al principio del siglo XXI, se acompaña de nuevas inflexiones que se identificarán a partir de tres ejemplos.
\end{abstract}

Palabras clave: Diario personal. Literatura francesa. Literatura contemporánea.

Abstract: Appeared in France at the end of the eighteenth century as a private form, the diary progressively became a literary genre during the

\footnotetext{
${ }^{1}$ Este artículo se inscribe en el marco del proyecto de investigación franco-español "Figures et frontières de l'intime à l'époque contemporaine / Figuras y fronteras de la intimidad en la época contemporánea" (OPE-2017-0042), Universidad de Pau et des Pays de l'Adour EFM (FR 4153), ALTER (EA7504), ITEM (EA 3002) en colaboración con el grupo de investigación "Lenguajes" (HUM 224) de la Universidad de Córdoba (España) y el laboratorio "Arts Plastiques" (EA 7472) de la Universidad de Rennes 2 (Francia). El trabajo ha sido revisado, en su traducción al español, por Álvaro Luque Amo, contratado FPU de la Universidad de Granada, y por Ana Armenta Deu, professeur agrégée de la Universidad de Pau y des Pays de l'Adour (Francia).
} 
nineteenth and twentieth century, and underwent various mutations at the end of the twentieth century. The trivialization of the genre at the beginning of the 21 st Century is accompanied by new inflections that will be identified on the basis of three examples.

Key Words: Diary. French literature. Contemporary literature.

\section{UN GÉNERO NUEVO}

A principios de este siglo XXI, el diario personal todavía es, en Francia, un género literario nuevo. Aparecido en los últimos años del siglo XVIII como una forma de escritura ordinaria, nacida de los registros de cuentas, de los diarios de viaje y de los diarios espirituales, el diario personal coloniza lentamente el campo literario durante el siglo siguiente. Esta lentitud es acentuada por la significativa diferencia temporal que existe entre las fechas de su redacción y su publicación: los primeros diarios son publicados únicamente tras la muerte de su autor, es decir, en algunos casos cuarenta años después del comienzo de su redacción.

Hay que esperar hasta la segunda mitad del siglo XIX para ver los diarios editados. Algunos diarios se publican de modo fragmentario a partir de 1850 (Maine de Biran, Eugénie de Guérin, Maurice de Guérin, Benjamín Constant) y luego en volúmenes completos después de 1880. En este momento, el género se va imponiendo en el paisaje editorial pero no sin polémicas y con diarios que, a menudo, no son de escritores literarios. Cabe citar, a parte del de Stendhal, los de Henri-Frédéric Amiel (profesor suizo), de María Bashkirtseff (pintora) o de Michelet (historiador).

A partir de esa época y hasta la Segunda Guerra Mundial, el diario personal empieza a ser utilizado como género literario por ciertos autores: Léon Bloy, es el primero en publicar el suyo volumen a volumen, a medida que los va escribiendo, en los últimos años del siglo XIX. André Gide, publica, en revistas, fragmentos del suyo, a principios del siglo XX. El género parece encontrar progresivamente un posicionamiento, muy al margen del campo literario, hasta el estallido de una nueva polémica, en 1939, con la publicación del Journal de André Gide en la colección de la Pléiade, la cual reúne únicamente obras literarias reconocidas: en este momento, la crítica comienza a plantearse si el diario se ha convertido 
realmente en un género literario.

Tras la Segunda Guerra Mundial, el número de diarios publicados aumenta de manera relevante: por una parte, porque numerosos textos del siglo XIX se publican de nuevo de forma más completa, a la vez que se publican autores de la primera mitad del siglo XX, pero también porque escritores todavía vivos publican el suyo, ya sean escritores reconocidos como François Mauriac, Henry de Montherlant, ya sea porque el diario es la gran obra de una vida (Paul Léautaud). Comienzan también a emerger diarios con una forma elaborada como una obra literaria. Citemos solo dos ejemplos: Toxique (1964), de Françoise Sagan (y Bernard Buffet para las ilustraciones), presenta la elaboración gráfica de su diario de desintoxicación; y Le Temps immobile (1974-88), de Claude Mauriac, es la recomposición de un diario inicial según una lógica que ya no es la de una sucesión temporal: el autor mezcla las entradas y las épocas para suscitar nuevas resonancias. Estas dos tentativas no tendrán la misma posteridad. Mientras que el diario se completa a veces con dibujos realizados por el mismo diarista o por un dibujante, la elaboración de Claude Mauriac no tuvo herederos, sin duda porque el dispositivo podía ser, en el mejor de los casos, solo imitado, lo que carece de interés literario.

\section{EL DIARIO A FINALES DEL SIGLO XX}

A finales del siglo $\mathrm{XX}$, la difusión del género es cada vez más amplia: se continúan publicando, de modo fiel y exhaustivo, los diarios personales del siglo XIX y de principios del siglo XX (Amiel, Gide), y se comienzan a publicar numerosos textos redactados durante la Segunda Guerra Mundial y hasta entonces inéditos. El diario acentúa también su integración en el campo literario mezclándose con la autobiografía retrospectiva, o enriqueciéndose con una dimensión lírica para componer diarios poéticos o libros de poemas fechados².

Diversas perspectivas de evolución se dibujan hasta constituir puntos de conexión para las prácticas literarias contemporáneas. El diario todavía se mantiene a veces secreto y como lista exclusiva de los acontecimientos, impresiones, sentimientos del día, revelados después de

${ }^{2}$ Sobre este punto, véase las reseñas "France 1945-197" y "France 1975-2015" del Dictionnaire de l'autobiographie: Écritures de soi de langue française (Simonet-Tenant, 2017). 
la muerte del autor ${ }^{3}$, pero se desarrolla también como una forma literaria que permite a su autor sacar partido de su poder de evocación referencial como un efecto de lectura. Para ello, los autores juegan con la presencia de lo realmente vivido, así como con la representación de la duración que propone la forma diarística, con o sin efecto lírico.

En efecto, a la vuelta del milenio ciertos autores imponen la forma diarística como la de la experiencia de lo cotidiano, de la experiencia existencial, de la experiencia amorosa y/o de la experiencia de escritura. De modo muy diferente, los diarios de Renaud Camus (30 volúmenes desde 1987), de Annie Ernaux (particularmente Se Perdre, 2001) o de Hervé Guibert (Le Mausolée des amants, 2001) se dedican a narrar una historia personal a semejanza de una novela, a construir los hilos de una historia como si fuera una novela ("soy un personaje de novela, desde el principio" Ernaux, 2001: 202), o, al menos, a buscar en las notas cotidianas "el texto de una novela" (Guibert, 2001: 38) .

Algunos autores comienzan también a publicar diarios como primera obra literaria, es decir a hacerse reconocer como autor por su diario. Charles Juliet ve rechazada una novela, L'Humiliation, antes de haber publicado su verdadero primer libro: su Journal 1957-1964 seguido rápidamente del volumen que abarca los años 1965 a 1968. André Blanchard, por su parte, adquiere cierta notoriedad con sus Carnets, y publica nueve volúmenes desde 1989 hasta su muerte. Esta publicación se produce tras el rechazo de la publicación de los otros textos: él proyectaba una entrada en la literatura mediante géneros de mayor reconocimiento, y las libretas de apuntes pueden ser percibidas como una estrategia alternativa después de varios fracasos. Estas publicaciones se realizan en pequeñas editoriales: POL (editor literario, en la editorial Hachette y posteriormente en su propia empresa editorial) en el caso de Juliet; Erti (editorial regionalista, en Vesoul, y posteriormente Le Diletante, editorial literaria bastante desconocida), en el caso de Blanchard.

La crítica universitaria no reconoció enseguida esta progresiva integración del diario en el campo de la literatura. Los estudios dedicados al género en los años 1960 y 1970 conciben el diario como una forma

\footnotetext{
${ }^{3}$ No teníamos, por ejemplo, conocimiento del diario de Belinda Cannone antes de que a ella se lo robaran y decidiese contar el trauma que le supuso el robo en un diario publicado, La Chair du temps (Cannone, 2012). Sobre este texto, véase Braud (2017).

${ }^{4}$ Sobre este texto, ver Braud (2002).
} 
complementaria a la obra literaria del autor, sin estar exactamente dentro de ella. Un crítico como Alain Girard, en 1963, asegura que "el diario íntimo se conformó como un género literario", y otra como Béatrice Didier, en 1976, señala que se trata "de un cierto tipo de escritura", para afirmar luego firmemente que "nada [...] parece más alejado de una obra que un diario" (Girard, 1986: viii) o que "no son 'obras' hablando con propiedad" (Didier, 1976: 7). Esta ambigüedad manifiesta la dificultad para reconocer el estatuto del género en el curso de estas décadas: los textos del siglo XIX y del principio del XX son más conocidos y los escritores comienzan a explotar las potencialidades literarias de la escritura diaria, pero esto no se relaciona con su constitución como género: "hablar de arte a propósito del diario íntimo sería pues un tipo de disparate", afirma de nuevo Alain Girard (1963: 598). Roland Barthes reformula por su parte la misma cuestión en 1979: “¿Puedo hacer del diario una 'obra’?” Para responder: "El diario no puede alcanzar el Libro (la Obra); es sólo Álbum” (1993: 424 y 435). No es casual que los primeros estudios universitarios que van a reconocer el diario como literatura se refieran a autores particulares y no al género de modo general, y a diaristas como Stendhal (Didier, 1983) ${ }^{5}$, cuyo diario directamente interroga la representación de sí en la vida cotidiana, o Gide (Marty, 1985), cuyo diario se ha impuesto como texto literario a mitad del siglo XX. Podemos observar también que un crítico tan agudo como Philippe Lejeune, gracias al cual la autobiografía fue reconocida en la investigación universitaria en los años 1970 y que se interesó por el diario a partir de 1990, contempla este último principalmente como una práctica y no estudia los diarios literarios. Así, como él mismo señala: "seguí dos pistas: tratar de descubrir diarios originales [y] interrogar a los productores de los diarios, haciendo encuestas" (2004). Solamente al principio del siglo XXI el diario ha adquirido un verdadero reconocimiento en los estudios universitarios, con el manual de Françoise Simonet-Tenant, Le Journal intime, genre littéraire et écriture ordinaire (2001) y el estudio de Michel Braud, La Forme des jours: pour une poétique du journal personnel (2006).

${ }^{5}$ El punto de vista de Didier ha cambiado entre 1976 et 1983. Escribe en Stendhal autobiographe: "El diario se crea; también crea un texto. [...] Se crea escribiendo y [...] al crearse, crea una obra" (1983: 101). 


\section{EL DIARIO AL PRINCIPIO DEL SIGLO XXI}

En estas primeras décadas del siglo XXI, el diario personal continúa labrándose una posición en el campo literario afirmando una continuidad de forma y desarrollando su capacidad para contar una historia. Tres ejemplos permiten ilustrar esta continuidad y esta transformación.

El primer texto es el más próximo a los diarios tradicionales por retomar las prácticas de escritura y las temáticas principales. JeanClaude Pirotte, poeta y prosista contemporáneo, autor de una cuarentena de libros, aparece en las historias de la literatura como un escritor que trenza la melancolía más emocionante y la trivialidad de la existencia ${ }^{6}$. Traverses (2017) es el diario de un año (junio de 2010 - junio de 2011) de un escritor que envejece (71 años) y está enfermo, presiente su próxima muerte (morirá en 2014), relata lo cotidiano (sus traslados, su dependencia al tabaco), sus recuerdos de infancia; presenta reflexiones amargas sobre el mundo, sobre la política francesa y más concretamente sobre la figura de Nicolas Sarkozy, por aquel entonces presidente de la República. Esta evocación se hace con un trasfondo de lecturas, particularmente de diarios (como el de Léon Werth escrito durante la Segunda Guerra mundial). Los ecos líricos son numerosos y el diario se convierte puntualmente en un compendio de fragmentos poéticos.

Traverses es un verdadero diario: un diario de enfermedad, de cólera contra el mundo contemporáneo y contra su violencia social; es un diario de atención poética a la cotidianidad, a la realidad y a su fantasmagoría. En esto, se inscribe en la tradición de los grandes diarios íntimos. No es un diario poético comparable a los que aparecieron a finales del siglo XX como Carnets de Philippe Jaccottet (1984-2001) o Journal du Saint-Laurent de Robert Marteau (1986), ni a la colección de poemas fechados presentados cronológicamente como el de Antoine Emaz (Os, 2004; Peau, 2008). La obra poética de J.-Cl. Pirotte se desarrolla en paralelo a la escritura del diario: al mismo tiempo que escribe su diario, Pirotte compone un libro de poemas, Cette âme perdue (2011), y evoca la corrección de las pruebas de dicho libro en su diario.

El segundo diario, el de Laurent Herrou, mezcla más las categorías. Este autor, nacido en 1967, ha publicado 4 novelas, 1 colección de cuentos

${ }^{6}$ Ver Viart (2005: 381); Touret (2008: 484); Bercot y Guyaux (1998: 861-2). 
y 5 volúmenes de diario. Herrou podría ser asimilado a los escritores que publican su diario después de haber sido reconocidos como novelistas, pero aparece más bien como un autor cuya obra está ampliamente contaminada por el diario. Su primera obra, Laura (2000), presentada por el autor como una novela, es muy parecida a una autobiografía y contiene más de cien páginas (2000: 153-262) del diario del autor. Laurent Herrou publica más tarde varios volúmenes de diario propiamente dicho: dos volúmenes motivados por una situación específica (una estancia en Montreal en 2012, una residencia en una escuela secundaria de enero a junio 2016) lo que limita su contenido en el tiempo, y a partir de 2015 tres volúmenes, uno por año, que siguen una lógica temporal.

Esta obra queda relativamente al margen del campo literario: la editorial del primer libro de Laurent Herrou (Balland) es conocida y reconocida, pero la colección en la que se publica Laura ("Le Rayon") es una colección concreta de LGBT y no de literatura general; las editoriales de los libros siguientes (H\&O, Jacques Flament) presentan una visibilidad mucho menor.

No obstante, el universo presentado en este diario aspira a ser un universo literario: Laurent Herrou menciona numerosos encuentros con escritores, proyectos y realizaciones con diversos artistas, y afirma estar en su elemento en un medio artístico (Herrou, 2016: 34-42). Se enorgullece de que su obra haya sido estudiada por un crítico especialista de la auto-ficción ${ }^{7}$. Este universo es también claramente reivindicado como homosexual: la vida de pareja, los diálogos, los conflictos, la insistencia sobre la dimensión sexual, la atención al riesgo de homofobia son temáticas recurrentes del diario que estructuran a la vez la personalidad del autor y la vida cotidiana narrada. Se trata también de un universo material relativamente al margen: Laurent Herrou vive en un castillo que pertenece a su abuela, en una aldea cerca de Bourges, en el centro de Francia, y evoca con regularidad sus dificultades financieras. Como otros diaristas antes que él (Léon Bloy, Renaud Camus), es pobre y está siempre sin blanca. Tampoco elude los momentos de angustia: "Cuando la soledad me grita en las orejas que tengo miedo, aúllo para sobrepasar su voz" (Herrou, 2016: 125) anota un día en el que su compañero se encuentra ausente. La expresión es a veces bastante coloquial e incluso grosera y denota

${ }^{7}$ Ver Arnaud Genon (2018). 
relaciones que pueden ser tensas como por ejemplo en este intercambio: "Mi abuelo dijo, prediciendo, a mi madre: espero que tengas una hija y que te jorobe tanto como tú me has jorobado a mí" (92). No obstante, el lector no conoce el detalle de estas relaciones ni la infancia del diarista.

El diario se sitúa en una encrucijada entre una confesión íntima siempre controlada, el deseo de ser reconocido como escritor y el sentimiento de saber escribir la vida cotidiana; y aún más: ser el único que sabe escribir sobre la vida cotidiana. Laurent Herrou reconoce tener bloqueos infantiles, sin explicitarlos, y evoca de vez en cuando las conversaciones con su psicoanalista. No es posible "decir todo" (38) en un diario, pero es un llamamiento al lector, a su mirada y a su atención. De hecho, el diarista interpela a su lector o sueña con ser leído después de su muerte: "Yo quisiera haber muerto y que usted tenga todo a disposición y lea este diario" (40).

La escritura del diario no nace ex nihilo. Los diarios se derivan de diferentes dispositivos de creación. La Part généreuse (2014) es el diario íntimo de un viaje a Quebec, en agosto de 2012, construido en relación con descripciones de fotografías; Autoportrait en Cher (et en mots) (2016) es el diario de una estancia del autor, escrito en la segunda persona del singular, en una escuela secundaria, entre enero y junio de 2016. En Journal 2015 (2016), es el dispositivo establecido por la editorial el que impulsa la escritura. En efecto, el editor, Jacques Flament, propuso al diarista publicar en línea, en su portal de internet, las anotaciones de su diario escrito a lo largo del año 2015 a medida que este lo escribía, comprometiéndose a publicar este diario en forma de volumen el año siguiente. No se trataba de redactar un blog más o menos íntimo, sino de emprender una aventura de escritura personal en público, programada para presentar un libro al cabo de un año. Para el diarista, la dificultad es múltiple: como ocurre en todo diario escrito para ser publicado, el diarista está confrontado con la contradicción de escribir y de verse escribir, pero debe también tener en cuenta la doble temporalidad derivada de una inmediata publicación en línea y otra en forma libresca el año siguiente. Y además debe contar con dos lecturas que se sobreponen la una a la otra: la del internauta que abre la página de la editorial y recorre un fragmento de la anotación del día, y la del lector de diario atento a la trama de la existencia del diarista. El reto es doble: frenar al internauta desatento y ofrecer al lector del libro una vida que se pueda seguir día a día, mes tras mes. 
No obstante, lo más significativo de esta empresa es el deseo de contar su existencia o, mejor dicho, de elaborar un libro que sea el relato de su existencia puesto que, al elaborar este relato, el diarista edifica "otra vida dentro de su propia vida, [...] la vida de uno" (Herrou y Genon, 2017: 34). El relato de los días es a la vez una construcción imaginaria (el relato proyectado de sí mismo), la imagen o una de las imágenes con la cual el diarista se identifica, y el rastro de un cotidiano que, aunque no totalmente ordinario, es por lo menos bastante banal. Por otra parte, la ausencia de elaboración estilística contribuye por otra parte a esta impresión de redacción común de una experiencia sin otra trama que la de una existencia como cualquier otra.

Dos conjuntos de publicaciones me permiten ilustrar la tercera orientación del género diarístico al principio del siglo XXI: el diario gráfico.

El diario gráfico hace su aparición en el paso del siglo XX al siglo XXI. El representante más visible es Joann Sfar, quien publica 12 volúmenes de libretas de apuntes de 2002 a 2015, con títulos evocadores ligados a su ocupación del momento, a una preocupación o a una pasión: Harmonica (2002), Ukulélé (2003), Missionnaire (2007), Si Dieu existe (2015), Si j'étais une femme je m 'épouserais (2016), por no citar más que algunos. En estos volúmenes, Sfar ofrece una continuidad de bosquejos rápidos, no reelaborados, tratados desde el punto de vista del diarista-dibujante o, más a menudo, del de un posible observador de dicho diarista. Los dibujos raramente están fechados, pero se presentan cronológicamente; integran burbujas, comentarios o relatos que se extienden en una o dos páginas: la imagen y el texto se completan tanto desde el punto de vista enunciativo como del narrativo.

Estas libretas de apuntes contienen anotaciones sobre la vida social, la vida profesional y la vida íntima del autor; reflexiones sobre su infancia, sobre sus amistades, sobre sus amores y sobre su vida familiar. Si j'étais une femme... está dedicado, por ejemplo, a la separación con su compañera y a la cura psicoanalítica que él empieza por este motivo. Estos textos son publicados al principio por L'Association, una editorial asociativa de jóvenes autores que producen cómics experimentales en blanco y negro, en libros muy cuidados y bastante caros, con un papel grueso y, en concreto, en una colección autobiográfica titulada "Côtelette".

Voyage aux îles de la Désolation, de Emmanuel Lepage (2011), 
ofrece una manifestación muy diferente de diario gráfico, aparecida unos años más tarde. Se trata en primer lugar de una historia original: un viaje a las Tierras australes y Antárticas francesas y más precisamente a las islas de Crozet, Kerguelen, Amsterdam y Saint-Paul, en el sur del océano indio. El espacio natural es hostil y los espectáculos naturales resultan excepcionales. El diario relata esta aventura traducida en dibujos, que comienza cuando el autor recibe la proposición de formar parte de una expedición de aprovisionamiento de estas islas, continúa en el barco, Marion Dufresne, que enlaza la isla de la Reunión con las otras islas, y luego en cada una de las islas visitadas, y concluye con su vuelta a la isla de la Reunión.

El diario publicado presenta la realidad tomada del ahora: las ilustraciones son realizadas, o por lo menos esbozadas, en el instante y reflejan la perspectiva del autor en ese preciso momento. Lo esencial de los dibujos está constituido por esbozos en lápiz que revelan una elaboración rápida y que permiten captar las posturas, el movimiento de las personas y del barco. Las ilustraciones más elaboradas son finalizadas y coloreadas después, y son presentadas rodeadas de bosquejos y de representaciones del contexto de su elaboración.

La intención estética es evidente y se percibe en el formato suficientemente grande del libro (33 x $24 \mathrm{~cm})$, en acuarelas por páginas dobles, en una variación continua de las perspectivas, y sobre todo en el juego entre el lápiz y el color, tanto en la portada como en el interior del libro, así como en la utilización recurrente de los colores negro, azul y verde. La sucesión de esbozos y acuarelas contribuye a la dinámica del relato y a la lírica de los instantes inmovilizados. Donde una fotografía del viaje, ubicada en la última página, atestigua de modo brutal la experiencia vivida, el dibujo y la acuarela hacen sentir la realidad y traducen la evanescencia de la atmósfera.

\section{PERSPECTIVAS DE EVOLUCIÓN}

En las dos primeras décadas del siglo XXI, el diario se constituye como género literario y experimenta diversas evoluciones. Sigue siendo una forma de escritura de sí y de lo cotidiano, sin aparente finalidad, es decir sin que el autor domine el fin de la historia y construya su historia con arreglo a una perspectiva conocida desde el principio de la redacción. 
Pertenece al canon literario — entró sin duda por la puerta pequeña- y permite la consideración del diarista como autor literario (Herrou y Genon, 2017: 19). Pero al mismo tiempo, por citar una expresión de Laurent Herrou, "el diario, es un puñetazo en el vientre. Es una bofetada, es un espejo que nos obliga a mirar" (Herrou y Genon, 2017: 23). El género expone la presencia de la realidad cotidiana sin borrar las asperezas de la experiencia.

Esta introducción del género en el espacio literario va acompañada de dos transformaciones progresivas. Por una parte, la elaboración de un diario como una obra, es decir, la publicación inmediata en volumen después de la redacción no induce a la eliminación de la vida cotidiana, material y amorosa, incluso en sus detalles más comunes o triviales. Este tipo de anotaciones que está presente en los diarios póstumos (como en el caso del diario de Jean-Claude Pirotte) se encontraba sólo de manera marginal en los diarios por entregas de autores vivos hasta los años 1980. Antes de esta fecha, el diarista no temía exponer su vida íntima tras su muerte, pero sí era más cuidadoso con lo que publicaba en vida. La segunda inflexión, con relación a los diarios de finales del siglo XX producidos en circunstancias comparables, es la ausencia de elaboración estilística. Mientras el diario de Renaud Camus, para citar un ejemplo muy visible en el campo literario de finales del siglo XX, juega sobre la elección, la variedad y la tonalidad del léxico, el de Laurent Herrou posee una gran neutralidad formal e incluso una banalidad real: el discurso ordinario se impone o tiende a imponerse como obra.

Por último, el desarrollo del diario gráfico está a la medida del emprendido por el cómic en el campo literario desde hace veinte o treinta años. Se ha convertido en una forma de narración de sí mismo o en la narración documental de una aventura. Hace unos años, podíamos leer reportajes dibujados a partir de las fotografías de un reportero ${ }^{8}$; ahora tenemos el propio dibujo del diarista-dibujante en situación. Voyage aux îles de la Désolation de Emmanuel Lepage ocupa con éxito este espacio estético.

Los Carnets de Joann Sfar parecen, no obstante, más interesantes desde el punto de vista de la confesión íntima. El autor inventa una forma, la libreta de apuntes dibujada en el curso de los días, bajo el modelo

${ }^{8}$ Véase Guibert, Lefèvre y Lemercier (2003). 
del diario, cuya diferencia estética se sitúa a la vez en el trazado y en la presentación gráfica distanciada, ligeramente humorística y autoirónica de la vida contada, en un contexto de interrogación existencial. Con $\mathrm{Si} \mathrm{j}$ 'étais une femme..., Sfar propone un recorrido de su historia personal, de su vida amorosa, de sus fantasmas... La intimidad se vuelve un objeto literario que integra la evocación de la infancia y de la vida amorosa, la reflexión sobre la existencia y sobre la escritura, y permite jugar con la proyección del lector.

En conclusión, comprobamos que una evolución se intuye: el diario tiende a instalarse en la creación literaria actual, incluso si es con modalidades de publicación más o menos marginales. Si, gracias a la publicación de diarios de escritores conocidos, el género fue objeto de un reconocimiento bastante ostensible durante los últimos años del siglo XX y a comienzos del siglo XXI (Charles Juliet, Renaud Camus, Annie Ernaux, Hervé Guibert), la producción actual parece menos sobresaliente en el conjunto de la producción literaria. En cambio, con las progresivas publicaciones en el paso del milenio, la creación literaria hace ahora del ordinario íntimo un objeto de relato, ya sea en el diario propiamente dicho (Pirotte, Herrou) o en el diario gráfico (Sfar). Esto no excluye otras perspectivas de desarrollo: la aparición del diario gráfico de reportaje (Lepage) muestra que las modalidades de exploración de las formas son diversas. Pero la permanencia de lo íntimo en el centro de la escritura de sí mismo, en el seno de escritores bastante jóvenes (40 años en el caso Laurent Herrou, 46 años en el de Joann Sfar), muestra que una nueva generación se apoderó de este formato, imprimiéndole su marca. La diferencia más significativa con relación a la generación anterior es su interés particularmente marcado por el relato de la vida cotidiana y ordinaria. Ahí donde los diarios de Juliet borran la evocación de la vida de cada día, donde los de Annie Ernaux o de Hervé Guibert hacen una aventura de la vida amorosa, donde el de Renaud Camus hace brillar la cotidianidad bajo la inteligencia de la mirada, el diario de Laurent Herrou recoge las huellas de los pequeños acontecimientos cotidianos mediante un lenguaje llano y sencillo. Y, si la representación de la realidad es por necesidad más compleja en el diario gráfico, podemos descubrir la misma atención en los detalles de la vida diaria en los Carnets de Joann Sfar y en cierto modo en el Voyage aux îles de la Désolation de Emmanuel Lepage. Si el diario ha conllevado desde hace más de dos siglos el discurso ordinario del diarista 
- pero este discurso era, hasta ahora, más sensible en los diarios póstumos que en los publicados por el autor-, tenemos la sensación de que, en lo sucesivo, este discurso será el propio de la obra literaria.

Podríamos pensar que tal evolución es la traducción directa de lo acontecido en la sociedad y que el diario solo anticipó la representación contemporánea de la existencia que se desarrolla, desde una quincena de años, en Internet. Las redes sociales presentan una "serie de rastros fechados" de la vida del autor, para repetir la definición mínima del diario propuesta por Philippe Lejeune y Catherine Bogaert (2006: 22). Las colecciones de fotos o vídeos en Facebook o Instagram, o las anotaciones de los blogs personales, se conforman como fragmentos de un cotidiano relato de sí. Incluso encontramos numerosos diarios íntimos en línea donde el autor cuenta — de modo anónimo - sus emociones amorosas o existenciales. Son historias individuales escritas día a día.

No obstante, la aproximación es demasiado rápida: no se debe confundir los usos de presentación del Yo en las redes sociales con el diario personal. El objetivo de la comunicación en línea es retener la atención del lector por la imagen presente del Yo; en el diario íntimo, es construir una historia de sí de quien el lector pueda informarse en el curso de una lectura continua. En el primer caso, el autor comunica el acontecimiento del día para obtener una recepción solo bajo la forma del "like"; en el segundo, el lector sigue la historia constituida por los acontecimientos narrados. En las redes sociales o los blogs, la historia es, en efecto, secundaria: hay que seguir a un autor regularmente y recordar los acontecimientos anteriores, o retroceder en la continuación de las fotos o las notas para conocer su historia; en el diario esta se constituye como el único objetivo: la vida se hace una historia. Las redes sociales ofrecen un retrato del autor en el instante; el diario ofrece el relato de su existencia a lo largo de los días.

Finalmente, el diario literario, cuyo movimiento se trató de sintetizar en el siglo XXI, tiene poco en común con las redes sociales. Si evoca el momento ordinario, es para hacer una historia de ello. Intenta contar una existencia común, universal, como la de un personaje literario, y a veces consigue hacerlo. 


\section{REFERENCIAS BIBLIOGRÁFICAS}

BARTHES, R. (1993). "Délibération". Le Bruissement de la langue: Essais critiques $I V$. Paris: Seuil.

BERCOT, M. et GUYAUX, A. (1998). Dictionnaire des lettres françaises: le XX $X^{e}$ siècle. Paris: Librairie Générale Française.

BLANCHARD, A. (1989-2018). Carnets [9 vols.]. Paris: Erti et Le Dilettante.

BRAUD, M. (2002). “Le texte d'un roman': Journal intime et fictionnalisation de soi". L'Esprit créateur XLII.4, 76-84.

(2006) La Forme des jours: pour une poétique du journal personnel. Paris: Seuil.

(2017). "Passé et présent dans le journal intime: lire le temps". Romanica Cracoviensia 17.2, 109-116 (también en http://www. ejournals.eu/Romanica-Cracoviensia/2017/Tom-17-\%20Numer-2 [11/04/2019]).

CANNONE, B. (2012). La Chair du temps. Paris: Stock.

DIDIER, B. (1976). Le Journal intime. Paris: PUF. (1983). Stendhal autobiographe. Paris: PUF.

ERNAUX, A. (2001). Se Perdre. Paris: Gallimard.

GENON, A. (2018). "En quête du je: les ego-territoires de Laurent Herrou". Relief - Revue électronique de littérature française 12.1, 113-124 (también en https://www.revue-relief.org [06/01/2019]).

GIRARD, A. (1963, reed. 1986). Le Journal intime. Paris: PUF.

GUIBERT, E. ; LEFEVRE, D. et LEMERCIER, F. (2003). Le Photographe. Paris: Dupuis.

GUIBERT, H. (2001). Le Mausolée des amants. Paris: Gallimard. HERROU, L. (2000). Laura. Paris: Balland. (2014). La Part généreuse. La Neuville-aux-Joûtes: Jacques Flament. (2016). Autoportrait en Cher (et en mots). La Neuville-aux-Joûtes: Jacques Flament. (2016). Journal 2015. La Neuville-aux-Joûtes: Jacques Flament. (2017). Journal 2016. La Neuville-aux-Joûtes: Jacques Flament. (2018). Journal 2017. La Neuville-aux-Joûtes: Jacques Flament.

HERROU, L. et GENON, A. (2017). L'Inconfort du je: Dialogue sur l'écriture de soi. La Neuville-aux-Joûtes: Jacques Flament.

JULIET, Ch. (1978a). Journal 1 1957-1964. Paris: Hachette. 
(1978b). Journal 2 1965-1968. Paris: Hachette.

LEJEUNE, Ph.(2004) "Projet d'enquête sur la pratique du journal personnel en Algérie". En L'Autobiographie en situation d'interculturalité, colloque de l'Université d'Alger, tome I, 31-49. Blida: Editions du Tell (también en http://www.autopacte.org/Enquete_Algerie.html [11/04/2019]).

LEJEUNE, Ph. et BOGAERT, C. (2006). Le Journal intime: Histoire et anthologie. Paris: Textuel.

LEPAGE, E. (2011). Voyage aux îles de la désolation. Paris: Futuropolis.

MARTY, E. (1985). L'Écriture du jour: le Journal d'André Gide. Paris: Seuil.

MAURIAC, Cl. (1974-1988). Le Temps immobile [10 vol.]. Paris: Grasset. PIROTTE, J.-Cl. (2017). Traverses. Paris: Le Cherche midi.

SAGAN, F. (1964). Toxique. Paris: Julliard.

SFAR, J. (2002). Harmonica. Paris: L'Association. (2003). Ukulélé. Paris: L'Association. (2007). Missionnaire. Paris: Delcourt. (2015). Si Dieu existe. Paris: Delcourt. (2016). Si j 'étais une femme je m'épouserais. Vanves: Marabout.

SIMONET-TENANT, F. (2001) Le Journal intime, genre littéraire et écriture ordinaire, Nathan.

(2017). Dictionnaire de l'autobiographie: Écritures de soi de langue française. Paris: Champion.

TOURET, M. (2008). Histoire de la littérature française du XXe s., t. II. Rennes: P.U. Rennes.

VIART, D. (2005). La Littérature française au présent. Paris: Bordas.

Recibido el 5 de abril de 2019.

Aceptado el 3 de junio de 2019. 